\title{
Activated sludge monitoring of a wastewater treatment plant using image analysis and partial least squares regression
}

\author{
A.L. Amaral ${ }^{\mathrm{a}, \mathrm{b}}$, E.C. Ferreira ${ }^{\mathrm{a}, *}$ \\ ${ }^{a}$ Centro de Engenharia Biológica, Universidade do Minho, Department of Biological Engineering, Campus de Gualtar, 4710-057 Braga, Portugal \\ ${ }^{\mathrm{b}}$ Escola Superior de Tecnologia e de Gestão, Instituto Politécnico de Bragança Campus de Santa Apolónia, Apartado 1138, 5301-854 Bragança, Portugal
}

Received 6 October 2004; received in revised form 21 December 2004; accepted 21 December 2004

Available online 24 January 2005

\begin{abstract}
The biomass present in a wastewater treatment plant was surveyed and their morphological properties related with operating parameters such as the total suspended solids (TSS) and sludge volume index (SVI). For that purpose image analysis was used to provide the morphological data subsequently treated by partial least squares regression (PLS) multivariable statistical technique. The results denoted the existence of a severe bulking problem of non-zoogleal nature and the PLS analysis revealed a strong relationship between the TSS and the total aggregates area as well as a close correlation between the filamentous bacteria per suspended solids ratio and the SVI.
\end{abstract}

(C) 2004 Elsevier B.V. All rights reserved.

Keywords: Environmental sciences; Activated sludge; Image analysis; PLS; Environmetrics

\section{Introduction}

The activated sludge is a complex ecosystem mainly of bacteria and protozoa. In fact, a good balance between the bacteria and protozoa and among the different species of each group is crucial for efficient pollution removal, good settleability properties and low suspended solids effluent levels. Bacteria agglomerate as aggregates, mainly due to exopolymers excretion and a filamentous bacteria backbone towards an ideal floc with reasonable size and well balanced floc-forming and filamentous bacteria composition. Furthermore, they should be also quite robust and have good settling capabilities, hence leading to a low organic matter and low turbidity final effluent. However, when the operating conditions are not perfect mainly in terms of organic load, nutrients and oxygen contents some malfunctions may occur such as the ones reported by Bitton [1]: pinpoint flocs bulking due to the absence of filamentous bacteria, leading to hardly settleable small flocs; filamentous bulking due to filamentous bacteria surplus, leading to hardly settleable huge and

\footnotetext{
* Corresponding author. Tel.: +351 253604 402; fax: +351 253678986 .

E-mail address: ecferreira@deb.uminho.pt (E.C. Ferreira).
}

linked flocs; dispersed growth due to floc-forming bacteria not flocculating leading a non-settling turbid effluent and zoogleal or viscous bulking due to flocs with poor settling abilities and compaction leading to high viscous and organic contents final effluents.

Image analysis has become nowadays a very important tool with a large field of applications due to its ability to remove the subjectiveness of human analysis, the possibility to extract quantitative data and avoid tedious and highly timeconsuming tasks to human researchers. Some of the most important applications of image analysis reside on medical imaging (magnetic resonance, X-ray computer tomography and ultrasound images), geographical data (landsat thematic and synthetic aperture radar based maps) and biology and biotechnology (muscle fibres and plant-cells embryo studies and DNA sequencing) among several others [2].

In fact, the rather tiring, imprecise and time-consuming classical methods to characterize aggregates and evaluate filamentous bacteria contents by manual counting under a microscope $[3,4]$ are being progressively step aside by the development of automated image analysis methods such as the works of Ganczarczyk [5], and Grijspeerdt and Verstraete [6] to characterize the aggregates morphology, and 
relate it to settleability properties. Indeed, automated image analysis seems to be an appropriate method to characterise quantitatively both aggregates and filamentous bacteria as shown by the methods proposed by Cenens et al. [7], Da Motta et al. [8] and Amaral et al. [9] subsequently used to monitor bulking events in pilot plants [10].

The prime objective of the present work resided on the survey of the filamentous bacteria and aggregates contents and morphology, in the biomass present in an activated sludge treatment plant, during a period of three and a half months. Furthermore, the relationship between biomass and operating parameters, such as the total suspended solids (TSS) and sludge volume index (SVI), could be enlightened by the use of the multivariable statistical technique partial least squares (PLS) regression.

\section{Materials and methods}

\subsection{Biomass sampling}

The activated sludge analyzed in this work, was taken from a wastewater treatment plant (WWTP) treating domestic effluents in Braga municipality for a period of three and a half months [11]. For the image analysis the maximum period of time between sample collection and image acquisition did not exceed $3 \mathrm{~h}$ with no more than half an hour without aeration. For the filaments and aggregates image acquisition, a volume of $50 \mu \mathrm{L}$ was taken to a slide and covered with a $24 \mathrm{~mm} \times 24 \mathrm{~mm}$ cover slip for visualization and image acquisition in phase contrast and direct light, respectively.

\subsection{Operating parameters}

The total suspended solids (TSS) were determined by weighting according to standard techniques [12]. The sludge volume index (SVI) was determined according to [13] as the volume in millilitres occupied by $1 \mathrm{~g}$ of a biomass suspension after $30 \mathrm{~min}$ settling. However, for highly concentrated mixed liquors it is preferable to use the diluted sludge volume index:

$\mathrm{SVI}=\frac{h_{30}}{h_{0} \mathrm{TSS}} f_{\mathrm{Dil}}$

where $h_{0}$ is the initial diluted sludge height, $\mathrm{h}_{30}$ the settled diluted sludge height after $30 \mathrm{~min}$ of settling time and $f_{\text {Dil }}$ the dilution factor.

\subsection{Image analysis}

\subsubsection{Image acquisition}

Filaments image acquisition (Fig. 1a) was accomplished through phase contrast microscopy on a Zeiss Axioscop microscope (Zeiss, Oberkochen) with a 100 times magnification and a total of 30 images per sample. Aggregates images (Fig. 1a) were acquired through the visualization on a
SZ 4045TR-CTV Olympus stereo microscope (Olympus, Tokyo) with a total magnification of 40 times and a total of 25 images per sample. In both cases the microscopes were coupled to a Sony CCD AVC-D5CE (Sony, Tokyo) grey scale video camera. Computer image grabbing was performed in 8 -bit ( 256 grey levels) $768 \times 576$ pixels matrix by a Data Translation DT 3155 (Data Translation, Marlboro) frame grabber using the commercial software package Image Pro Plus (Media Cybernetics, Silver Spring).

Aggregates and filamentous bacteria morphological descriptors were determined using two programs created in Matlab 6.5 (The Mathworks, Natick): the floc program and the filaments program. Each programme consists of 2 sub-routines that can be used together or separately. A more detailed description of each program code as well as the algorithms used to identify the filamentous bacteria or aggregated biomass and measure the main morphological parameters can be found in [10,9]. The first of the sub-routines is an image processing automatic procedure to obtain the binary images of the filaments from the original 256 grey level images. The second one is a fully automatic image analysis programme to determine and save the morphological parameters of the filaments/aggregates. The main stages of the first programme comprise the image pre-treatment, image segmentation and debris elimination whereas the second programme is oriented to the morphological parameters determination.

\subsection{Filaments program}

\subsubsection{Image pre-treatment}

The first step of the filaments programme resides on the normalisation and improvement of the grey level original image. In this stage the original image is divided by a background image to minimize background light differences, re-shifted back to retain the original median, followed by a grey scale cut off (value 2 ) and finally a $4 \times 4$ pixels grey scale bottom hat filter [17] is applied in order to improve the filaments definition.

\subsubsection{Aggregates segmentation}

This stage consists primarily in the segmentation of the aggregates and elimination of these in the enhanced filaments image. Prior to the aggregates elimination a series of steps consisting on eliminating filaments and filament-like debris is performed by the summation of the grey scale cut off image. Subsequently the image is enhanced by the absolute difference of the image to a value of 0.5 , a 10 th order grey scale closing followed by an aggregates segmentation with a pre-defined aggregates threshold value being carried out. At the end of this stage, an aggregates gap filling is performed, as well as the elimination of aggregates and debris smaller than $10 \times 10$ pixels from the enhanced filaments image by a 10th order erosion and a reconstruction operation. 

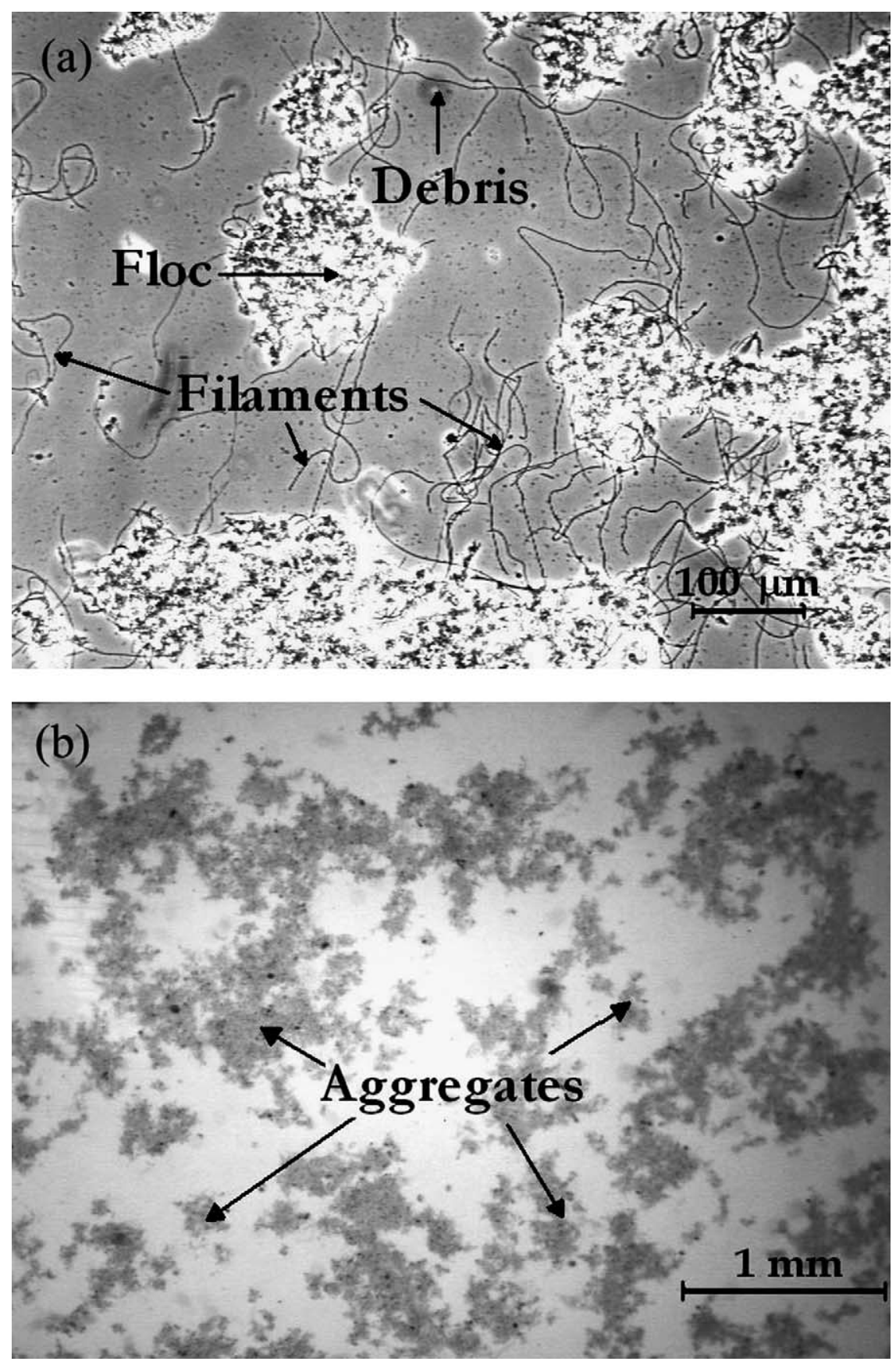

Fig. 1. Filamentous bacteria (a) and aggregates (b) images.

\subsubsection{Filaments segmentation}

This stage consists primarily in a pre-processing step followed by the segmentation of the filaments. The preprocessing step resides on the determination of the aggregates to image area ratio (A/I ratio) percentile and the elimination of the pixels with lower values than the found percentile, followed by the difference to the grey scale cut off image in order to enhance the image. Finally the segmentation step is performed with a pre-defined filaments threshold value.

\subsubsection{Debris elimination}

This step is achieved by the determination and use of a filaments marker image in the filaments and filament-like debris image. The filaments marker image can be defined as an image containing at least one pixel belonging to the filaments of interest and none of the filament-like debris. The determination of a second percentile value based on both pre-defined filaments segmentation percentile level and the $\mathrm{A} / \mathrm{Iratio}$, followed by an images conjugation and a reconstruction operation are used for that purpose. The 
filaments marker image is then used as a mask in order to perform a reconstruction of the real filaments in the resulting filaments segmentation image. Finally the determination of each object area and gyration radius [14], allowed the elimination of debris with area smaller than 32 pixels or gyration radius smaller than 1.2. The last step of this stage consists in attributing a different intensity value for each of the different filaments (labelling) found in the binary image.

\subsubsection{Morphological parameters determination}

The main morphological parameters determined by this programme are: total filamentous bacteria number (Fil Nb) as the number of end-points, divided by 2 and rounded upwards, after a 12 pixel length branch deletion; filamentous bacteria mean length $(L)$ as the sum of the pixels of the filament skeleton multiplied by a factor of 1.1222 [15] to account for the different directions, divided by the number of filaments; total filamentous bacteria length (TL) as the sum of all the filaments skeleton pixels within an image multiplied by a factor of 1.1222 [15].

\subsection{Flocs program}

\subsubsection{Image pre-treatment}

The first step of the flocs programme resides on the normalisation and improvement of the grey level original image. In this stage the original image is first divided by a background image to minimize background light differences and then equalized by histogram equalization in order to enhance the contrast of the aggregates boundaries. Subsequently the image is filtered by a Wiener filter [3] so that pixel noise can be softened.

\subsubsection{Aggregates segmentation}

In the first stage of the segmentation step an aggregates boundary image is obtained by the difference between a first order grey scale dilation and a first order grey scale erosion images. Afterwards a two-step aggregates segmentation was achieved with the determination and use of an aggregates marker image obtained by image segmentation at a 0.075 threshold level as well as a preliminary aggregates image by image segmentation at a pre-defined threshold level. The aggregates marker image (image containing at least one pixel belonging to the aggregates of interest and none of the debris) is then used as a mask in order to perform a reconstruction of the real aggregates in the preliminary aggregates image. Seemingly are performed an aggregates gap filling and a second segmentation procedure based on the determination of the aggregates to image area ratio (A/I ratio) percentile. The pre-treatment Wiener filter image is then segmented by this percentile threshold level and conjugated (only common white pixels remaining) with the resulting binary image from the first segmentation procedure.

\subsubsection{Debris elimination}

The first step of this stage consists on aggregates gap filling and an exclusion operation (only pixels which are simultaneously white in the first image and black in the second image remaining) with the resulting image from the aggregates segmentation stage. Subsequently the elimination of aggregates and debris smaller than $6 \times 6$ pixels by a third order erosion and a reconstruction operation is performed. Finally the determination of each object area allowed the elimination of debris with area smaller than a pre-defined aggregates minimum size, as well as objects cut off by the image boundaries. The last step of this stage consists in attributing a different intensity value for each of the different filaments (labelling) found in the binary image.

\subsubsection{Morphological parameters determination}

The main morphological parameters determined are the following: total aggregates number (Floc $\mathrm{Nb}$ ) as the total number of aggregates not cut off by the images boundaries; aggregates mean area $(A)$ as the sum of the pixels of the projected surfaces of the aggregates, divided by the number of aggregates; total aggregates area (TA) as the sum of all the aggregates pixels within an image; extent (Ext) defined as the ratio between the area of an object and its bounding box area [16]; eccentricity (Ecc) calculated using the area $(A)$ and the second order moments $\left(M_{2}\right)$ of an object by [2]: $E \mathrm{cc}=\frac{(4 \pi)^{2}\left(M_{2 X}-M_{2 Y}\right)^{2}+4 M_{2 X Y}^{2}}{A^{2}} ;$ convexity (Conv) defined as the ratio between the convex perimeter and the perimeter of an object [2]; roundness (Round) calculated using the area $(\mathrm{A})$ and the convex perimeter $\left(P_{\text {Conv }}\right)$ of an object by [2]: Round $=\frac{4 \pi A}{P_{\mathrm{Conv}}{ }^{2}}$; compactness (Comp) calculated using the area $(A)$ and the maximum Feret diameter $\left(F_{\text {Max }}\right)$ of an object by [17]: Comp $=\frac{\sqrt{(4 / \pi) A}}{F_{\operatorname{Max}}} ;$ solidity (Sol) defined as the ratio between the object area and the convex envelope area [17].

With the aggregates mean area, aggregates total area, filamentous bacteria length and total filamentous bacteria length data, three new parameters could be determined: filamentous bacteria length per aggregates mean area ratio $(L / A)$ calculated as the ratio between the filamentous bacteria length and the aggregates mean area; filaments contents versus aggregates contents (TL/TA) calculated as the ratio between the total filamentous bacteria length and the total aggregates area; and filaments contents versus solids contents (TL/TSS) calculated as the ratio between the total filamentous bacteria length and the total suspended solids.

\subsection{Partial least squares regression (PLS)}

Partial least squares regression (PLS), is an iterative algorithm that extracts linear combinations of the essential features of the original $\mathbf{X}$ data while modelling the $\mathbf{Y}$ data dependence on the work set being therefore well suited for multivariate calibration. The most important advantage of this method reports to the non-problematic handling of multicollinearities relying on an iterative algorithm, which makes 
possible the treatment of data with more features than objects [18].

In this method, the latent variables $u_{i}$ (matrix $\mathbf{U}$ ) are used for modelling the objects separately in the matrix of $\mathbf{Y}$ dependent data, whereas, the $t_{i}$ variables (matrix $\mathbf{T}$ ) are used for modelling the objects separately in the $\mathbf{X}$ matrix of independent data. The latent variables $\mathbf{U}$ and $\mathbf{T}$ are the basis of the regression model and are determined by $\mathbf{U}=\mathbf{A} \times \mathbf{T}+\mathbf{E}$ (PLS components matrix $\mathbf{A}$ and error matrix $\mathbf{E}$ ) in an iterative process with the centred matrices of $\mathbf{X}$ and $\mathbf{Y}$ as starting points [18].

Simca 7.01 (Umetri, Umëa) software package was used to perform PLS analysis from the data set. This software iteratively computes one PLS at a time, that is, one vector each of $\mathbf{X}$-scores $(t), \mathbf{Y}$-scores $(u)$, weights expressing the correlation between $\mathbf{X}$ and $\mathbf{U}(w)$, weights expressing the correlation between $\mathbf{Y}$ and $\mathbf{T}(c)$ and loadings $(p)$. The PLS components are calculated in descending order of importance. For the response variables $(m)$ in $\mathbf{Y}$, the multiple correlation coefficient $\left(R^{2} \mathbf{Y}_{\text {cum }}\right)$ or goodness of fit is given by [19]:

$$
R^{2} \mathbf{Y}_{\text {cum }}=\sum R^{2} \mathbf{Y}_{a}
$$

where $R_{2} \mathbf{Y}_{a}$ is the sum of squares of all the $\mathbf{Y}$ 's explained by each extracted component $(a)$.

The variable importance in the projection (VIP) represents the influence of each parameter $(k)$ of the data matrix $(\mathbf{X})$ on the results matrix $(\mathbf{Y})$, so that the parameters with a VIP larger than 1 have an above average influence on the result and are, therefore, the most relevant for explaining Y. SIMCA computes the variable importance in the projection (VIP) as the sum over all model dimensions (PLS components) of the variable influence $\mathrm{VIN}_{\mathrm{ak}}$ [19]:

$\mathrm{VIN}_{k}=\sum \mathrm{VIN}_{a k}^{2}$

For a given PLS dimension ( $a), \mathrm{VIN}_{a k}^{2}$ is equal to the squared PLS weight $w_{a k}^{2}$ of that parameter, multiplied by the percent explained sum of squares by that PLS dimension $\left(R^{2} \mathbf{Y}_{a}\right)$. The variable importance is then normalized so that $\sum(\mathrm{VIP})^{2}$ equals the number of the data parameters $(K)$. The parameters

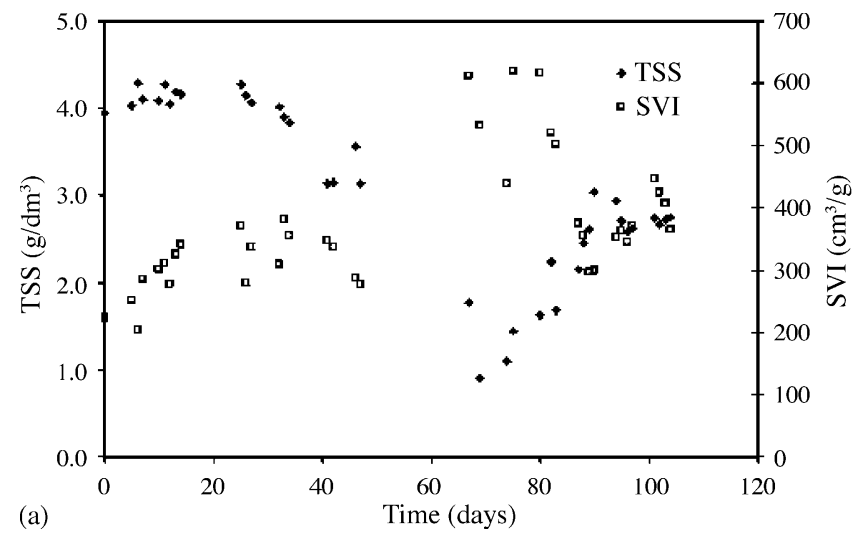

that are found to be the most important are the ones presenting a VIP value larger than 1 [19].

The overall error of each predicted value $\hat{y}_{i}$ can be given by the normalized distance $\hat{y}_{\operatorname{Dist}(i)}$ to the model [19]:

$\hat{y}_{\operatorname{Dist}(i)}=\frac{\sqrt{\sum f_{\mathrm{im}}^{2} /(K-A)}}{\sqrt{\sum \sum f_{\mathrm{im}}^{2} /\left(N-A-A_{0}\right)(M-A)}}$

where $\left(N-A-A_{0}\right)$ is the number of the degrees of freedom for PLS, $K$ and $M$ are, respectively, the number of $\mathbf{X}$ data parameters and $\mathbf{Y}$ response variables, $A$ the number of PLS components, $A_{0}$ is 1 or 0 , depending on whether $\mathbf{Y}$ is centered or not and $f_{\text {im }}$ are the $\mathbf{Y}$ residuals for each observation $(i)$ and response variable $(m)$.

The method used to discard the outliers was based on the calculation of the normalized distance $\left(\hat{y}_{\text {Dist }(i)}\right)$ to the model and elimination of the data points (days) presenting a distance value larger than 1 [19].

\section{Results}

In this work, the aggregates contents and morphology from Braga WWTP were surveyed, for a period of three and a half months, by the aggregates number and projected area and the above mentioned morphological parameters whereas the free filamentous bacteria contents was surveyed by the filamentous bacteria length and number. Overall, approximately 140,000 aggregates were studied in this survey and the main collected data is summarized in Fig. 2 and Table 1.

Throughout all the survey time the sludge volume index (SVI) presented values quite high ranging from 200 to $620 \mathrm{~cm}^{3} / \mathrm{g}$, implying thus the existence of a severe bulking problem. Furthermore, this situation was more problematic in the period between days 67 and 87 with values normally higher than $500 \mathrm{~cm}^{3} / \mathrm{g}$. The total suspended solids contents (TSS) were somewhat higher than the normal operating limits ranging from 0.5 up to $4.5 \mathrm{~g} / \mathrm{dm}^{3}$, with the lower values corresponding to the higher values of the SVI between days 67 and 87 .

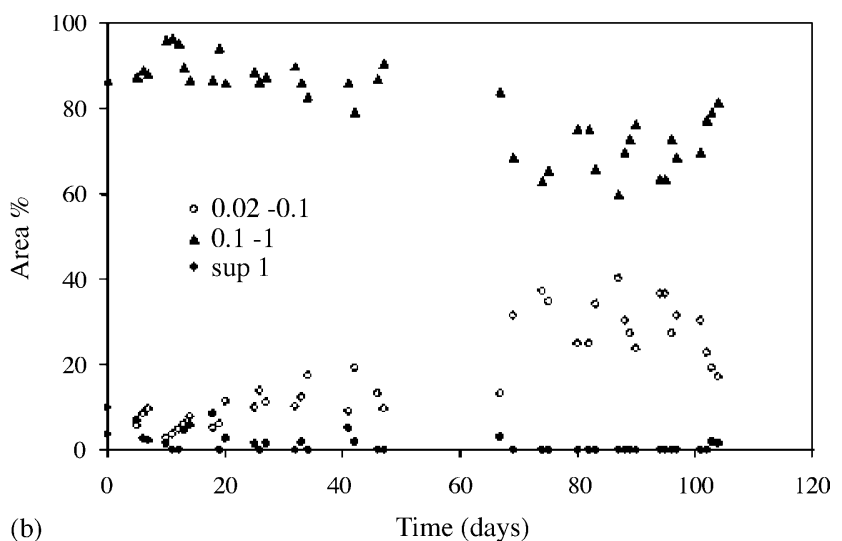

Fig. 2. Parameters behavior: (a) SVI and TSS; (b) aggregates area distribution. 
Table 1

Main morphological parameters average for each aggregates class

\begin{tabular}{llll}
\hline & Convexity & Eccentricity & Solidity \\
\hline $0.02-0.1$ & 0.93 & 0.77 & 0.71 \\
$0.1-1$ & 0.61 & 0.80 & 0.65 \\
$>1$ & 0.27 & 0.85 & 0.57 \\
\hline
\end{tabular}

The analysis of the aggregates area distribution clarifies the nature of the flocs within the aeration tank. The significant presence of the $0.1-1 \mathrm{~mm}$ diameter aggregates with respect to the $0.02-0.1 \mathrm{~mm}$ and to the $>1 \mathrm{~mm}$ diameter aggregates seems to point out the predominance of normal flocs instead of pin point flocs or zoogleal flocs. Furthermore, the large zoogleal flocs were practically inexistent from day 46 until the end of the survey. Therefore, the referred bulking problems within the aerated tank could not be of zoogleal nature, but probably of filamentous nature as pointed out by the coincidence between the higher SVI values and the smaller aggregates predominance.

With respect to the morphological parameters from the seven parameters studied, three (see Table 1) emerged representing the three major aspects of the aggregates morphology: aggregates ability to take the lesser possible place (solidity), aggregate elongation (eccentricity) and aggregate edges roughness (convexity). From this data it was apparent the formation of somewhat loose elongated structures with the smaller aggregates presenting smooth edges differing from the rougher edges of the larger aggregates.

Also, two parameters originating from the filaments contents versus aggregates contents (TL/TA) in one hand and from the filaments contents versus solids contents (TL/TSS) on the other were included due to its importance on the prediction of the SVI (Fig. 3). From the analysis of these two parameters the most significant information that could be withdrawn is the fact that there was a strong peak in both parameters correlating roughly with the peak found for the SVI. Therefore, a straight dependence of the SVI with the relationship between the free filamentous bacteria and aggregates ratio should be considered leading towards the ex- istence of a filamentous bulking phenomenon. Furthermore, the TL/TSS values larger than $10,000 \mathrm{~mm} / \mathrm{mg}$ [2] clearly indicate the existence of a filamentous bulking problem.

In order to enlighten the parameters relationships the PLS regression was performed to this data set with the TSS and SVI as $\mathbf{Y}$ variables and the morphological parameters as $\mathbf{X}$ variables.

Regarding the PLS analysis in what the SVI is concerned, five major aspects were studied: free filamentous bacteria contents (total filamentous bacteria length and number and filaments contents versus aggregates contents); free filamentous bacteria characterization (filamentous bacteria mean length and filaments mean length versus aggregates mean area); aggregates contents (total aggregates area and number); aggregates size (aggregates mean area) and aggregates morphology (convexity, compactness, extent, solidity, roundness and eccentricity). The parameters most suited for representing each of these major aspects were found to be, respectively, TL/TA, $L / A$, TA, $A$, and convexity. With respect to the PLS analyses for the TSS only three key aspects were studied: free filamentous bacteria contents; aggregates contents and aggregates morphology. The parameters most suited for representing each of these major aspects were found to be, respectively TL/TA, TA and convexity.

When the PLS regression of the raw data was performed within SIMCA with a built-in autofit option, no significant improvement in the prediction ability occurred for more than one latent variable in the TSS study attaining a value for the multiple correlation coefficient (goodness of fit) of 0.822 . Regarding the SVI study a two latent variables PLS regression was found to be appropriate, as the use of more latent values shown no significant improvement, resulting in a multiple correlation coefficient of 0.741 .

The variable importance in the projection (VIP) between the TSS and SVI and their key aspects are explicited in Table 2. The parameter found to contribute the most for the TSS (highest VIP value) was the one representing the aggregates contents key aspect, namely the total aggregates area (TA) with a value of 1.112 (sole value larger than 1). With respect to the SVI, the filaments contents versus aggregates
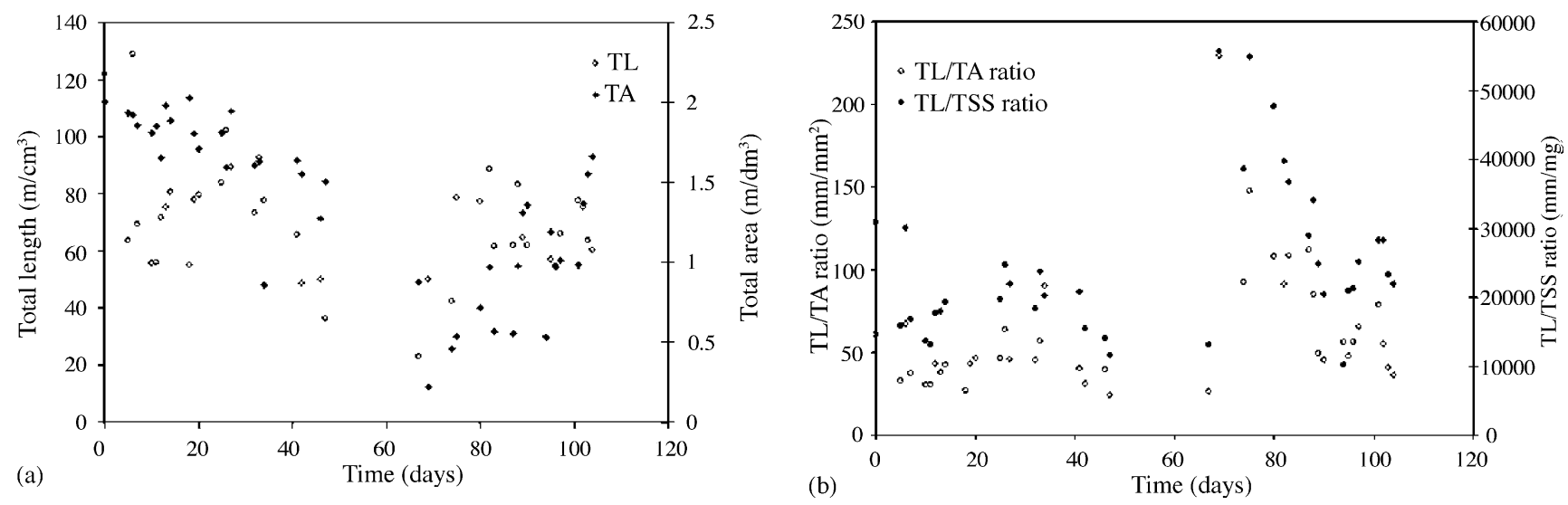

Fig. 3. Aggregates and filamentous bacteria contents (a) and TL/TA and TL/TSS behavior (b). 
Table 2

Partial least squares regression variable importance (VIP) for TSS and SVI with key parameters

\begin{tabular}{lll}
\hline & TSS & SVI \\
& (1 latent variable) & (2 latent variables) \\
\hline TL/TA & 0.901 & 1.412 \\
TA & 1.112 & 0.893 \\
Convexity & 0.976 & 0.900 \\
L/A & - & 0.896 \\
Area & - & 0.773 \\
\hline
\end{tabular}

contents (TL/TA) representing the free filamentous bacteria contents key aspect was, by far, contributing the most with a value of 1.412 (sole value larger than 1).

The results were obtained after discarding the days 34 and 67 for TSS and 0, 6, 26, 67, 69 and 87 for SVI, as suggested by a first PLS trial using all the data set. The method used to discard the outliers was based on the calculation of the normalized distance $\hat{y}_{\text {Dist }(i)}$ to the model and elimination of the data points (days) presenting a distance value larger than 1. Furthermore, these outliers were shown to be set quite apart from the trendline formed by all the other points when plotted in a predicted values versus observed values graph upon the first PLS trial.

With respect to the result of the PLS analysis for the SVI, the parameter found to contribute the most (TL/TA) presented a correlation regression of 0.839 . However, the ob-
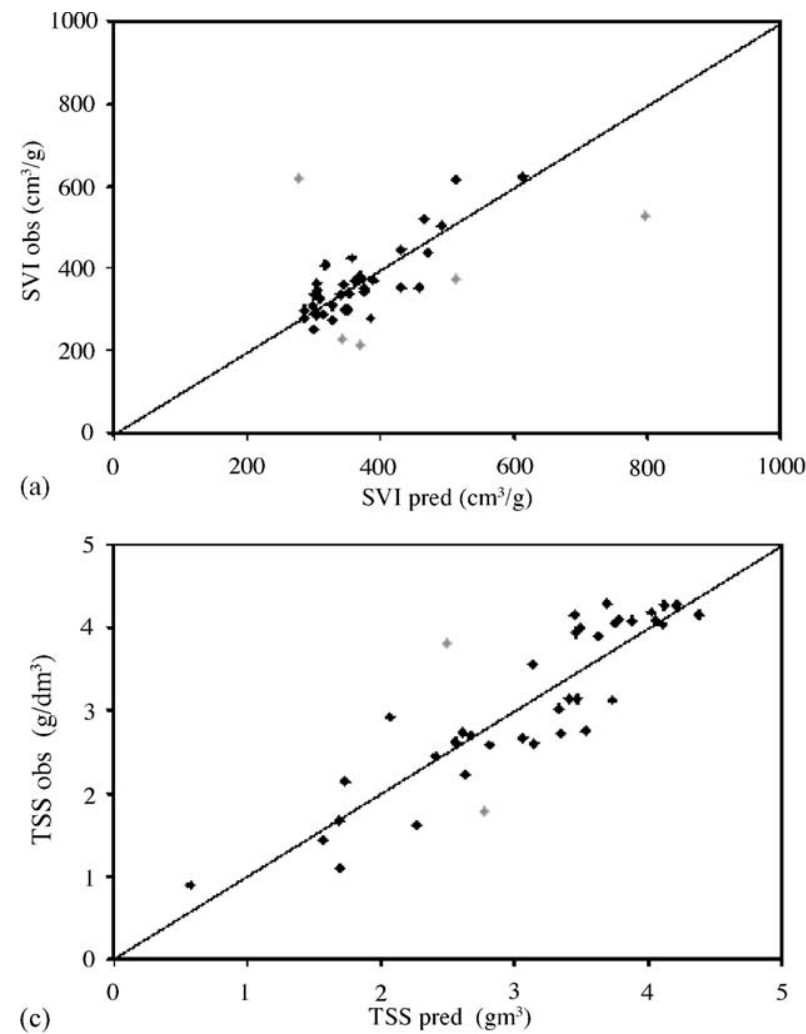

served versus predicted SVI correlation regression value of 0.861 (Fig. 4a) was not satisfactory, despite of a good agreement (9.9\% difference) between the observed and predicted values.

The strong relationship between the SVI and TL/TA parameter led to the investigation of another parameter (TL/TSS) partially determined by image analysis and partially by physical measurements. Doing so, when the SVI values were plotted directly against this parameter (Fig. 4b), a 0.885 correlation regression was found with only 3 rejected days (in opposition to the 6 days rejected by the PLS). Consequently this parameter seemed to provide the best assessment of the SVI values and could be used, at some extent, to monitor the SVI behaviour in a wastewater treatment plant aeration tank.

Regarding the PLS analysis between the TSS and the image analysis data emphasized the relationship with the aggregates contents (TA) with a 0.934 correlation regression (Fig. 4c) quite satisfactory considering the fact that only 2 days were discarded (same as the PLS). Therefore, it seemed reasonable to infer that the TSS behaviour could be satisfactory monitored by the TA parameter. Once again a linear relationship involving the above-mentioned key aspects of the TSS was tested and the observed versus predicted TSS correlation regression was found to be 0.906 lower than the relationship between the TSS and the TA parameter (Fig. 4d). Furthermore, the observed and predicted TSS values differed
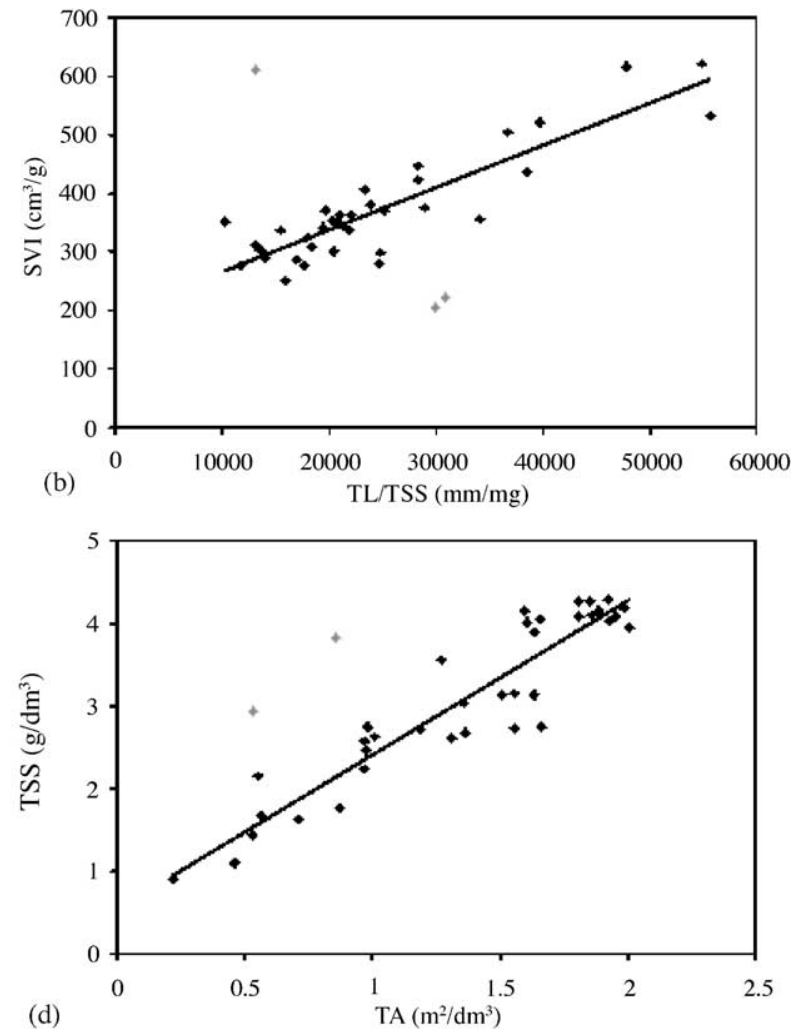

Fig. 4. Correlations between the observed and predicted SVI (a), between SVI and TL/TSS (b), between the observed and predicted TSS (c), and between TSS and TA (d), (grey points discarded). 
from each other $13.1 \%$. Hence, and given the satisfactory correlation regression for the relationship between the TSS and TA it was found preferable to use only this parameter to monitor the TSS contents.

\section{Discussion and conclusions}

From the beginning of the experiment and throughout all the survey time very high sludge volume index (SVI) values were detected, ranging from 200 to $620 \mathrm{~cm}^{3} / \mathrm{g}$, denoting the existence of a severe bulking problem. Regarding the total suspended solids (TSS) contents their values fell a little higher than the normal operating limits between 0.5 and $4.5 \mathrm{~g} / \mathrm{dm}^{3}$, with the lower values corresponding to the period of SVI higher values.

From the aggregates morphological analysis it was apparent the formation of somewhat loose elongated structures with the smaller aggregates presenting smooth edges differing from the rougher edges of the larger aggregates.

The aggregates area distribution showed meaningless values for the larger aggregates throughout the survey, in opposition to the clear predominant $0.1-1 \mathrm{~mm}$ class, which seems to point out the prevalence of normal flocs instead of pinpoint or zoogleal flocs. This fact allows inferring that the experienced bulking problems within the aerated tank were probably not of zoogleal nature and rather of a filamentous one. This hypothesis is further emphasised by the time overlap of the higher SVI period and the smaller aggregates predominance period in the survey.

Furthermore, this activated sludge monitoring experiment allowed retrieving some important conclusions with respect to the relationships between the aggregates contents and morphology and the free filamentous bacteria contents on one hand and the SVI and the TSS on the other. Analysing the filaments versus solids content (TL/TSS) and the filaments versus aggregates content (TL/TA), a strong resemblance between the SVI and these two parameters behaviour points was found pointing towards the existence of a filamentous bulking phenomenon. Furthermore, the TL/TSS values larger than $10,000 \mathrm{~mm} / \mathrm{mg}$ clearly indicate the existence of a filamentous bulking problem.

Regarding the partial least squares regression (PLS) in what the SVI is concerned, the predicted and observed SVI values 0.861 correlation regression (after 6 days discarded) was found to be lower than the 0.885 correlation regression, when plotted directly against the TL/TSS parameter (for only 3 rejected days). One may therefore assume that the TL/TSS parameter may be used, at some extent, to monitor the SVI behaviour in a wastewater treatment plant aeration tank.

With respect to the PLS analysis for the TSS the parameter that was found to contribute the most was the aggregates contents (TA) with a quite satisfactory correlation regression of
0.934 for 2 days discarded. Consequently it seems realistic to infer that the TSS could be satisfactory monitored by the TA parameter, even more attending to the corresponding correlation regression of 0.906 between the predicted and observed TSS.

Given the fact that, in the course of this survey, the SVI values were always quite high, only the relationships for high SVI values could be studied, whereas for a wastewater treatment plant working with satisfactory SVI values these relations may not stand true. Furthermore, as no points were present in the lower section of the regression lines the search of good correlation values may also have been hindered.

\section{Acknowledgements}

Fundação para a Ciência e a Tecnologia (Portugal) provided financial support for A.L. Amaral through a doctoral research grant (PRAXIS XXI/BD/20325/99). The authors express their gratitude to R. Pereira (AGERE, EM) and S. Rodrigues for their cooperation.

\section{References}

[1] G. Bitton, Wastewater Microbiology, Wiley/Liss, New York, 1994.

[2] C.A. Glasbey, G.W. Horgan, Image Analysis for the Biological Sciences, Wiley, Chichester, 1995.

[3] M. Sezgin, Water Res. 16 (1982) 83-88.

[4] J.C. Palm, D. Jenkins, D.S. Parker, J. Water Pollut. Contr. Feder. 54 (1980) 1087.

[5] J.J. Ganczarczyk, Water Sci. Technol. 30 (1994) 87-95.

[6] K. Grijspeerdt, W. Verstraete, Water Res. 31 (1997) 1126-1134.

[7] C. Cenens, R. Jenne, J.F. Van Impe, Water Sci. Technol. 45 (2002) 85-91.

[8] M. Da Motta, M.N. Pons, N. Roche, H. Vivier, Biochem. Eng. J. 9 (2001) 165-173.

[9] A.L. Amaral, S. Rodrigues, M. Mota, E.C. Ferreira, Proceedings of the 2nd IASTED International Conference on Visualization Imaging and Image Processing, Málaga, 2002, pp. 300-305.

[10] A.L. Amaral, Image analysis in biotechnological processes: applications to wastewater treatment, Ph.D. Thesis, Braga, 2003.

[11] S. Rodrigues, Bulking Filamentoso: Análise, Diagnóstico, Soluções, Industrial Training Report, Braga, 2000.

[12] G. Tchobanoglous, F.L. Burton, Metcalf and Eddy's Wastewater Engineering: Treatment Disposal and Reuse, McGraw-Hill, New York, 2003.

[13] APHA, AWWA, WPCF, Standard Methods for the Examination of Water and Wastewater, American Public Health Association, Washington, DC, 1989.

[14] M.N. Pons, H. Vivier, Adv. Biochem. Eng./Biotechnol. 66 (1999) $133-184$.

[15] A.E. Walsby, A. Avery, J. Microbiol. Meth. 26 (1996) 11-20.

[16] The Mathworks, Matlab Documentation Set, CD-ROM, 2002.

[17] C.R. Russ, The Image Processing Handbook, CRC Press, Boca Raton, 1995

[18] J.W. Einax, H.W. Zwanziger, S. Geiss, Chemometrics in Environmental Analysis, VCH Verlagsgesellshaft, Weinheim, 1997.

[19] Umetri AB, User's Guide to SIMCA, CD-ROM, 1998. 